\title{
Population Relationship of Three Forestry Species, Aegiphila ferruginea, Oreopanax ecuadorensis, Vallea stipularis; in the Leonan de Llucud Forest, Chambo Canton, Chimborazo Province
}

Memories II International Congress Forests and Agroforestry for the 21st Century

Corresponding Author:

O. Guadalupe Arias

oguadalupe@espoch.edu.ec

Published: 21 January 2021

Production and Hosting by Knowledge E

(c) O. Guadalupe Arias et al. This article is distributed under the terms of the Creative Commons Attribution License, which permits unrestricted use and redistribution provided that the original author and source are credited.

\section{Relación Poblacional de Tres Especies Forestales, Aegiphila ferruginea, Oreopanax ecuadorensis, Vallea stipularis; en el Bosque Leonan de Llucud, Cantón Chambo, Provincia de Chimborazo}

\section{O. Guadalupe Arias, K. Chávez Inca, and J. Rodríguez Guerra}

Escuela de Ingeniería Forestal, Facultad de Recursos Naturales, Escuela superior politécnica de Chimborazo

\section{Abstract}

The present investigation aims to relate the population of three native forest species of the Andean region of Ecuador: Oreopanax ecuadorensis Seem, Aegiphila ferruginea, and Vallea stipularis in the Leonan de Llucud high montane evergreen forest located in the Chambo canton. Through the inventory and observation of the distribution of these three species in three altitudinal floors, establishing ten plots within which circular subplots of five meters radius were placed, taking the species under study as the center, all individuals with diameter at breast height greater than or equal to $7 \mathrm{~cm}$ were selected. For each altitudinal floor, the Importance Value Index, the Simpson and Shannon-Weaver Biodiversity Indices, and the Sorensen Similarity Index were calculated. The results presented below are the result of the data obtained in the field and its interpretation. $V$. stipularis and O. ecuadorensis Seem show a close relationship, while $A$. ferruginea has a low population due to the exploitation it is subjected to in this forest, thus asserting the presence of this species in the red book at a near-threatened level.

Keywords: evergreen forest, native forest species, altitudinal floors.

\section{Resumen}

La presente investigación pretende relacionar la población de tres especies forestales nativas de la región andina del Ecuador Oreopanax ecuadorensis Seem, Aegiphila ferruginea y Vallea stipularis en el bosque siempre verde montano alto Leonan de Llucud ubicado en el cantón Chambo; mediante la inventariación y observación de la distribución de estas tres especies en tres pisos altitudinales, estableciendo diez parcelas dentro de las cuales se colocaron subparcelas circulares de cinco metros de radio tomando como centro a las especies en estudio, se seleccionaron todos los individuos con Diámetro a la Altura del Pecho, mayor o igual a $7 \mathrm{~cm}$. Para cada piso altitudinal se calculó el Índice de Valor de Importancia, los Índices 
de biodiversidad de Simpson y Shannon - Weaver y el Índice de similitud de Sorensen. Los resultados que a continuación presentamos son resultado de los datos obtenidos en campo y su interpretación. Teniendo que $V$. stipularis y $O$. ecuadorensis Seem presentan gran afinidad; en tanto que $A$. ferruginea presenta una baja poblacional debido a la explotación a la cual es sometida en este bosque, aseverándose así la presencia de esta especie en el libro rojo en nivel de casi amenazado.

Palabras Clave: bosque siempre verde, especies forestales nativas, pisos altitudinales.

\section{Introducción}

Entre las bondades ambientales que los bosques nos ofrecen destacamos que son por excelencia los pulmones del planeta. En el catálogo de Plantas Vasculares del Ecuador se han registrado 9865 especies en la región andina representando el 64,4\% del total [1]. Los remanentes de ecosistemas nativos que persisten en los paisajes fragmentados constituyen una herramienta valiosa para la preservación de especies y comunidades nativas [2].

Las estimaciones para la deforestación en el periodo 2013-2018 muestran una tendencia hacia una disminución y establece un nivel de 55.000 ha de deforestación [3]; a esto se suma el hecho de que en un ecosistema todos y cada uno de sus componentes y poblaciones interactúan en varios grados. Obteniendo especies que se adaptan de manera corporal o de comportamiento y en ciertos casos de manera genética obteniendo nuevas especies, distintas a sus antecesores [4]. La presente investigación pretende contar con información actualizada y oficial sobre los recursos forestales presentes en el bosque Leonan de Llucud.

\section{Materiales y Métodos}

\subsection{Materiales}

\subsubsection{Ubicación geográfica}

La presente investigación se realizó en la Provincia de Chimborazo, Cantón Chambo, parroquia La Matriz, en el bosque primario Leonan de Llucud, perteneciente a la Asociación San Pedro de Llucud (Figura 1). Con coordenadas $01^{\circ} 43^{\prime} \mathrm{S}$ de latitud y $78^{\circ} 33^{\prime} \mathrm{W}$ de longitud a una altitud: 3200 m.s.n.m.

\subsubsection{Características climáticas}

Precipitación: 500 a 1000 mm/año

Temperatura: 10 a $18^{\circ} \mathrm{C}$ 


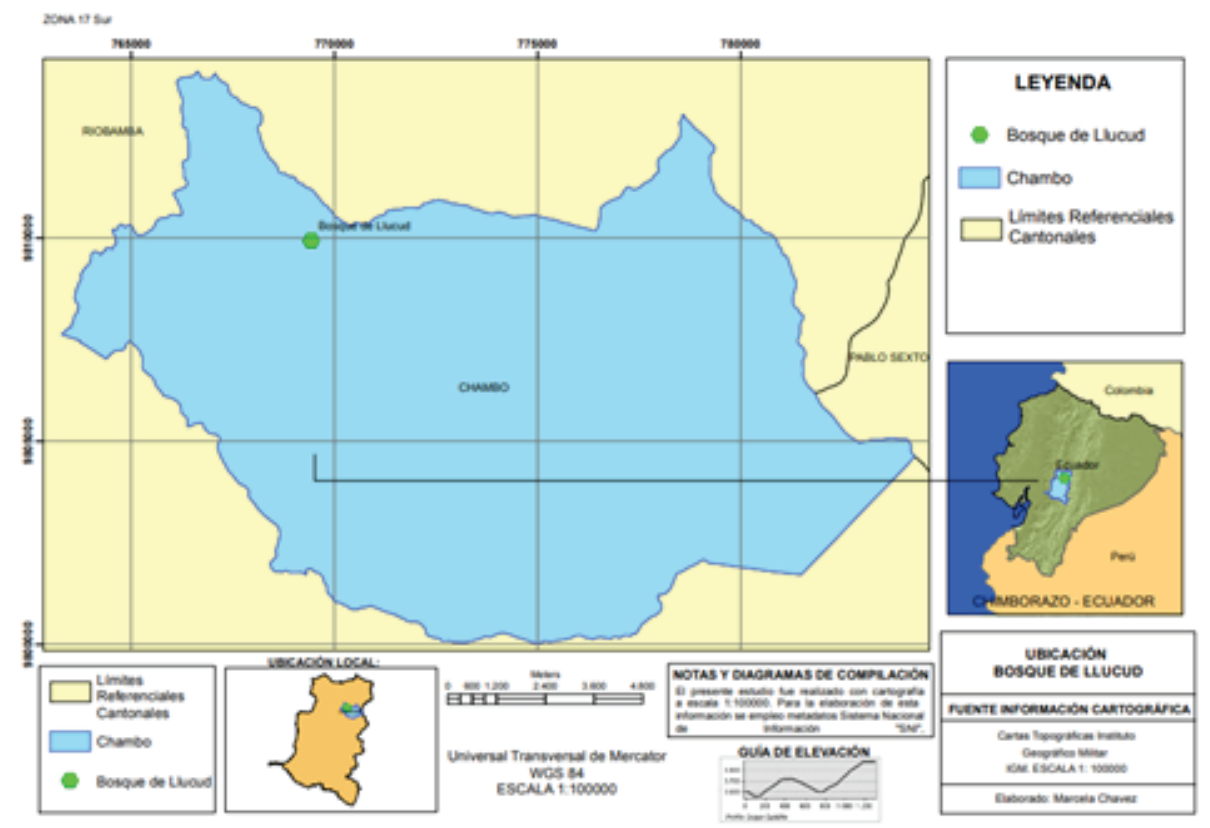

Figure 1

Ubicación geográfica del bosque Leonan de Llucud.

Según [5], pertenece a la clasificación ecológica de Bosque Siempreverde montano alto.

\subsubsection{Materiales de campo}

Lápiz, libreta de campo, borrador, cinta métrica, piola, pintura en spray, forcípula.

\subsubsection{Equipos}

GPS, cámara fotográfica, flash memory, hipsómetro computadora, impresora.

\subsubsection{Materiales en el herbario}

Papel periódico, hilo, carpeta.

\subsection{Métodos}

\subsubsection{Reconocimiento y delimitación del área de estudio}

El reconocimiento y delimitación se realizó el día 01 de junio de 2018, mediante un recorrido del bosque y la toma de coordenadas con un GPS marca Garmin y la ubicación de marcas para seccionar los pisos altitudinales obteniendo el piso altitudinal A que 
comprende desde los 3300 a 3400 m.s.n.m.; el piso altitudinal B que comprende desde los 3400 a 3500 m.s.n.m. y el piso altitudinal C que va desde los 3500 a 3550 m.s.n.m.

\subsubsection{Toma de datos}

El trabajo de campo se realizó los días 17 y 29 de julio de 2018. Con un área de muestreo de $1000 \mathrm{~m}^{2}$ segmentados en 10 parcelas; tres para el piso altitudinal A, cuatro para el piso altitudinal $B$ y tres para el piso altitudinal $C$ con un intervalo de sistematización de $200 \mathrm{~m}$. Dentro de cada parcela se colocaron subparcelas circulares de cinco metros de radio $\left(78.5 \mathrm{~m}^{2}\right)$, tomando como centro las especies objeto de estudio. Se tomó el DAP y la altura de las especies con un diámetro mayor o igual a $7 \mathrm{~cm}$. Se colectaron muestras de todos los individuos marcados posterior a la obtención del permiso de recolección de flora en el Ministerio del Ambiente de la ciudad de Riobamba.

Las muestras fueron prensadas y posteriormente llevadas para su identificación en el Herbario de la Escuela Superior Politécnica de Chimborazo (CHIEP) a nivel de familia, género y especie. Para mayor información de revisó el Catálogo de plantas vasculares.

\subsubsection{Interpretación de los datos de campo}

Con los datos obtenidos se realizó la aplicación de las siguientes fórmulas recomendadas por [6].

1. Índice de valor de importancia

Abundancia: Está representada por el número de individuos de todas las especies

Área basal: $\pi^{*}(\mathrm{DAP})^{2} / 4$

Densidad Relativa:

$$
\mathrm{DR}=\frac{\# \text { de individuos de una especie }}{\# \text { total de individuos en la parcela }} * 100
$$

Dominancia Relativa:

$$
\mathrm{DMR}=\frac{\text { Área basal de la especie }}{\sum \text { del área basal de todas las especies }} * 100
$$

Índice de Valor de Importancia:

$$
\mathrm{IVI}=\frac{\mathrm{DR}+\mathrm{DMR}}{2}
$$

2. Índice de diversidad de Simpson

$$
\text { IDS }=1-\sum(\mathrm{Pi})^{2}
$$

donde: IDS: Índice de Diversidad de Simpson; Pi: Proporción de individuos.

3. Índice de diversidad de Shannon-Weaver

$$
\mathrm{H}=-\sum\left[\mathrm{pi}^{*} \log (\mathrm{pi})\right]
$$

donde: pi: (ni/n) Proporción del número total de individuos; ni: Número de individuos de una misma especie; $n$ : Número total de individuos. 
4. Índice de similitud de Sorensen

$$
\text { ISS }=\frac{2 C}{A+B},
$$

donde: ISS: Índice de similitud de Sorensen; A: Número de especies del muestreo 1; B: Número de especies del muestreo 2; C: Número de especies en común de los 2 muestreos.

Con los resultados obtenidos luego de la aplicación de fórmulas se realizó el análisis de las subparcelas por especie para cada piso altitudinal.

\section{Resultados}

En la (Tabla 1) correspondiente al inventario realizado en el bosque Leonan de Llucud se obtuvieron 78 individuos, los cuales pertenecen a 8 familias, 11 géneros y 11 especies. Obteniendo un mayor número de especies en el piso altitudinal $C$ con un $62 \%$, en tanto que el piso altitudinal que presento menor número de individuos fue el piso altitudinal B con $15 \%$.

\section{Table 1}

Listado de especies registradas en el bosque Leonan de Llucud por piso altitudinal.

\begin{tabular}{|c|c|c|c|}
\hline Familia & Género & Especie & No. de Ind. \\
\hline \multicolumn{4}{|l|}{ Piso altitudinal $\mathbf{A}$} \\
\hline Araliaceae & Oreopanax & Oreopanax ecuadorensis Seem & 3 \\
\hline Asteraceae & Gynoxys & Gynoxys sp. & 4 \\
\hline Asteraceae & Verbesina & Verbesina sp. & 2 \\
\hline Elaeocarpaceae & Vallea & Vallea stipularis & 3 \\
\hline Melastomataceae & Brachyotum & Brachyotum ledifolium & 3 \\
\hline Primulaceae & Myrsine & Myrsine andina & 3 \\
\hline \multicolumn{4}{|l|}{ Piso altitudinal B } \\
\hline Araliaceae & Oreopanax & Oreopanax ecuadorensis Seem & 8 \\
\hline Elaeocarpaceae & Vallea & Vallea stipularis & 4 \\
\hline \multicolumn{4}{|l|}{ Piso altitudinal C } \\
\hline Araliaceae & Oreopanax & Oreopanax ecuadorensis Seem & 2 \\
\hline Asteraceae & Aristiguietia & Aristiguietia glutinosa & 6 \\
\hline Asteraceae & Gynoxys & Gynoxys sp. & 22 \\
\hline Columelliaceae & Collumelia & Collumelia oblonga & 3 \\
\hline Elaeocarpaceae & Vallea & Vallea stipularis & 3 \\
\hline Ericaceae & Maclenia & Maclenia cordifolium & 3 \\
\hline Melastomataceae & Miconia & Miconia bracteolata & 7 \\
\hline Solanaceae & Solanum & Solanum venosum & 2 \\
\hline TOTAL & & & 78 \\
\hline
\end{tabular}

No. de individuos: Número de individuos. 
En la (Tabla 2) se observa que los tres pisos altitudinales presentan una dominancia de la especie Gynoxys sp. El piso altitudinal que presenta mayor área basal es el C con 2,8 esto debido a que en este se encontró el mayor número de individuos adultos.

\section{Table 2}

Índice de valor de importancia de las especies por piso altitudinal en el bosque Leonan de Llucud.

\begin{tabular}{|c|c|c|c|c|c|c|}
\hline \multirow[t]{2}{*}{ Familia/Especie } & \multicolumn{2}{|c|}{ P. altitudinal A } & \multicolumn{2}{|c|}{ P. altitudinal B } & \multicolumn{2}{|c|}{ P. altitudinal C } \\
\hline & No. de ind. & IVI & No. de ind. & IVI & No. de ind. & IVI \\
\hline \multicolumn{7}{|l|}{ ARALIACEAE } \\
\hline Oreopanax ecuadorensis & 3 & 19,09 & 8 & 64,62 & 2 & 4,24 \\
\hline \multicolumn{7}{|l|}{ ASTERACEAE } \\
\hline Gynoxys sp. & 4 & 17,67 & 0 & 0,00 & 22 & 45,61 \\
\hline Verbesina $s p$ & 2 & 9,45 & 0 & 0,00 & 0 & 0,00 \\
\hline Aristiguietia glutinosa & 0 & 0,00 & 0 & 0,00 & 6 & 9,59 \\
\hline \multicolumn{7}{|l|}{ COLUMELLIACEAE } \\
\hline Collumelia oblonga & 0 & 0,00 & 0 & 0,00 & 3 & 4,24 \\
\hline \multicolumn{7}{|l|}{ ELAEOCARPACEAE } \\
\hline Vallea stipularis & 3 & 19,51 & 4 & 35,38 & 3 & 8,62 \\
\hline \multicolumn{7}{|l|}{ ERICACEAE } \\
\hline Maclenia cordifolium & 0 & 0,00 & 0 & 0,00 & 3 & 6,27 \\
\hline \multicolumn{7}{|l|}{ MELASTOMATACEAE } \\
\hline Brachyotum ledifolium & 3 & 15,42 & 0 & 0,00 & 0 & 0,00 \\
\hline Miconia bracteolata & 0 & 0,00 & 0 & 0,00 & 7 & 16,19 \\
\hline \multicolumn{7}{|l|}{ PRIMULACEAE } \\
\hline Myrsine andina & 3 & 18,85 & 0 & 0,00 & 0 & 0,00 \\
\hline \multicolumn{7}{|l|}{ SOLANACEAE } \\
\hline Solanum venosum & 0 & 0 & 0 & 0,00 & 2 & 5,24 \\
\hline TOTAL & 18 & 100 & 12 & 100 & 48 & 100 \\
\hline
\end{tabular}

No. de ind: Número de individuos; IVI: Índice de valor de importancia.

Para el piso altitudinal A de 3300 a 3400 m.s.n.m. se obtuvo 18 individuos pertenecientes a 5 familias, 18 géneros y 18 especies. Teniendo que la especie más abundante en este piso altitudinal es G. sp., perteneciente a la familia Asteraceae con 4 individuos y un IVI de 17, 67; mientras que Verbesina $s p$. (Asteraceae) presento el menor número de individuos teniendo así 2 individuos con un IVI de 9,45; cabe mencionar que esta especie se limitó únicamente a este piso altitudinal.

Para el piso altitudinal B de 3400 a 3500 m.s.n.m. se obtuvo 12 individuos pertenecientes a 2 familias, 2 géneros y 2 especies. La especie más abundante es Oreopanax ecuadorensis Seem (Araliaceae) con 8 individuos y un IVI de 64,62 mientras que Vallea stipularis (Elaeocarpaceae) presenta 4 individuos con un IVI de 35,38 .

Para el piso altitudinal C de 3500 a 3550 m.s.n.m. se obtuvo 49 individuos pertenecientes a 7 familias, 8 géneros y 8 especies. La especie G. $s p$. (Asteraceae) es 
la que presento mayor número de individuos con 22 y un IVI de 45,61; en tanto que Solanum venosum (Solanaceae) presento el menor número de individuos con 2 y un IVI de 5,24 limitándose a este piso altitudinal.

En el cálculo del Índice de Diversidad de Simpson (Tabla 2) por piso altitudinal de obtuvo que el piso altitudinal A registró un mayor Índice de diversidad con un valor de 0,83; seguido por el piso altitudinal $C$ que presento un valor de 0,74 y finalmente el piso altitudinal $\mathrm{B}$ que presento un valor de 0,44 . Con estos valores podemos mencionar que la diversidad florística en el piso altitudinal $A$ y $C$ es alta según lo menciona [7].

Según el índice de diversidad Shannon-Weaver (Tabla 2) se obtuvo que el piso altitudinal $A$ presenta un valor de 1,77; seguido del piso altitudinal $C$ que presenta un valor de 1,68 y el piso altitudinal $B$ un valor de 0,64 . Con estos valores podemos emitir que el piso altitudinal $A$ y $C$ con valores de 1,77 y 1,68 respectivamente registran una diversidad media, en tanto que el piso altitudinal B con un valor de 0,64 presenta una diversidad baja según lo menciona [8].

\section{Table 3}

Índices de biodiversidad de Simpson y Shannon-Weaver para cada piso altitudinal.

\begin{tabular}{l|ll} 
Piso altitudinal & I. B. Simpson & I. B. S-W \\
A & 0,83 & 1,77 \\
B & 0,44 & 0,64 \\
C & 0,74 & 1,68
\end{tabular}

I. B. Simpson: Índice de biodiversidad de Simpson; I. B. S-W: Índice de biodiversidad de Shannos-Weaver.

Al realizar el índice de similitud de Sorensen según [6] se obtuvo que para los pisos altitudinales $\mathrm{A}$ y $\mathrm{B}$ el índice de similitud es del 13\% con 2 especies en común; para los pisos altitudinales $A$ y $C$ la similitud es del $9 \%$ con 3 especies en común y para los pisos altitudinales $\mathrm{B}$ y $\mathrm{C}$ el índice de similitud es del 7\% con 2 especies en común. Al obtener 2 y 3 especies compartidas entre las comparaciones realizadas en los tres pisos altitudinales y al obtener porcentajes bajos para el índice de similitud de Sorensen podemos mencionar que existe un bajo índice de similitud; lo cual puede deberse a factores de sitio como la altitud, esto en conjunto con el aprovechamiento de especies que se realiza, sobre todo en los pisos altitudinales más bajos y con menos pendiente lo cual facilita el acceso a los mismos.

En el piso altitudinal B se encontró individuos de Aegiphila ferruginea, mismos que han sido cortados, se los identifico por los rebrotes que estos presentaban por lo que se puede emitir que esta especie es sometida a una explotación por parte de las personas que viven en los alrededores logrando casi la pérdida total de esta especie en el bosque en estudio.

\section{Discusión}

Vallea stipularis (Elaeocarpaceae), se encontró en los tres pisos altitudinales (Tabla 1) con una distribución baja de individuos adultos que pasan los $10 \mathrm{~cm}$ de DAP, suponiendo 
que esta especie es característica de este tipo de bosque. Así también lo corrobora [9]; Oreopanax ecuadorensis Seem (Araliaceae), se encontró en los tres pisos altitudinales con un número más significativo de individuos que pasan los $10 \mathrm{~cm}$ de DAP. Estos resultados difieren con [9] quien menciona que está especie únicamente se registró a los 3435 m.s.n.m. La frecuencia del resto de especies se limita a uno o dos gradientes altitudinales.

Se puede aludir con este inventario que la presencia de especies en un piso altitudinal, pero en otro no, depende de la combinación de varios factores como: Suelo, pendiente, altitud, humedad, entre otras. Así lo menciona [10], la distribución de especies está definida principalmente por la altitud a través de cambios en la temperatura, suelo y precipitación.

La observación de $A$. ferruginea cerca de Oreopanax ecuadorensis Seem nos señala la afinidad que presentan estas dos especies así lo menciona [11] mismo que utilizó estas dos especies en la implementación de un corredor biológico en un bosque montano alto al norte del Ecuador.

Según [9-12] la diversidad de especies es inversamente proporcional a la altitud, encontrando una disminución de especies a medida que aumenta el piso altitudinal lo cual difiere con nuestros resultados obtenidos, teniendo que para el piso altitudinal C que es el más alto presenta un mayor número de individuos, en tanto que el piso altitudinal $\mathrm{B}$ es el que presenta menor número de individuos; esto es un indicativo del aprovechamiento al cual es sometido el bosque por parte de las personas que se encuentran en los alrededores ya que los pisos altitudinales más bajos presentan mayor facilidad de acceso

\section{Conclusiones}

En este estudio se obtuvieron un total de 78 individuos, los cuales pertenecen a 8 familias, 11 géneros y 11 especies. Obteniendo un mayor número de especies en el piso altitudinal $\mathrm{C}$ con un $62 \%$ del total. Mientras que el piso altitudinal que presento menor número de individuos fue el piso altitudinal B con $15 \%$ del total.

La especie G. sp. fue la que registro mayor dominancia en dos pisos altitudinales (A, C) con 26 individuos y 63,28 de IVI.

Según el Índice de Diversidad de Simpson el piso altitudinal A y C la diversidad florística tienden a ser homogénea presentando valores cercanos a 1, el piso altitudinal B presenta una diversidad florística baja ya que su valor se aleja de la unidad.

El Índice de Diversidad de Shannon-Weaver se observa que el piso altitudinal A y $C$ presentan una diversidad media con valores de 1,77 y 1,68 ,respectivamente mientras que el piso altitudinal $C$ presenta una diversidad baja con 0,64.

De acuerdo con el Índice de Similitud de Sorensen al realizar las comparaciones de los tres pisos altitudinales presentan una baja similitud obteniendo que el porcentaje más alto de similitud es del $13 \%$ entre el piso altitudinal A y el piso altitudinal B con 2 especies en común. 
Las especies en estudio $V$. stipularis y $O$. ecuadorensis presentan gran relación con especies pertenecientes a la familia Asteraceae en los pisos altitudinales $\mathrm{A}$ y $\mathrm{C}$.

De acuerdo con el análisis poblacional en el piso altitudinal $B, V$. stipularis y $O$. ecuadorensis presentan la mayor afinidad entre ellas.

La especie $A$. ferruginea se encuentra en peligro de desaparecer en el bosque Leonan de Llucud, encontrando solamente individuos con rebrotes esto se atribuye a la explotación por parte de las personas de los alrededores.

\section{References}

[1] Jørgensen P, Neill D, León S. Catálogo de las plantas vasculares de Ecuador. Monographs in systematic botany from the Missouri Botanical Garden; 1999. Spanish

[2] Arroyo V, Pineda E, Escobar F, Benítez J. Value of small patches in the conservation to plant-species diversity in highly fragmented rainforest. Veracruz, México: Instituto de Ecología; 2009.

[3] Ministerio del Ambiente de Ecuador. Plan Nacional de Restauración Forestal [National Forest Restoration Plan]. 2014-2017. Quito, Ecuador: MAE; 2014. Spanish

[4] Gispert C. El mundo de la ecología [The world of ecology]. Barcelona, España: Océano; 2001. Spanish

[5] Sierra R. Propuesta preliminar de un sistema de clasificación de vegetación para el Ecuador Continental [Preliminary proposal for a vegetation classification system for Continental Ecuador.]. Quito, Ecuador: INEFAN/GEF-BIRF y EcoCiencia; 1999. Spanish

[6] Cerón C. Distribución de murciélagos (Chiroptera) a través de un gradiente altitudinal en las estribaciones orientales de los Andes ecuatorianos [Distribution of bats (Chiroptera) through an altitudinal gradient in the eastern foothills of the Ecuadorian Andes,Internet]. $1^{\circ}$ ed. Quito; 2003 [citado el 12 de septiembre de 2018]. Disponible en: www.puce.edu.ec. Spanish

[7] Schaffner S, Pascal M, Otto R, Delgado J, Arévalo J, Fernández J. Ecología, estructura y dinámica de las poblaciones de la sabina canaria (Juniperus turbinata ssp. canariesis) en Tenerife y La Gomera [Ecology, structure and dynamics of the populations of the Canarian juniper (Juniperus turbinata ssp. Canariesis) in Tenerife and La Gomera]. Tenerife, España: Universidad de La Laguna; 2005. Spanish

[8] Tirira D, Boada C. Diversidad de mamíferos en bosques de Ceja Andina alta del nororiente de la provincia de Carchi [Diversity of mammals in the upper Andean Ceja forests of the northeastern province of Carchi]. Quito, Ecuador: ESPE; 2009. Spanish

[9] Cuvi M. Estudio de la diversidad florística a diferente gradiente altitudinal en el bosque Montano alto Llucud, cantón Chambo, provincia de Chimborazo [Study of floristic diversity at different altitudinal gradients in the upper Llucud Montane forest, Chambo canton, Chimborazo province,Tesis de grado. Ingeniero Forestal]. Riobamba, Ecuador: Escuela Superior Politécnica de Chimborazo; 2010. Spanish

[10] Zacarias Y. Composición y estructura del bosque templado de Santa Catarina Ixtepeji, Oaxaca, a lo largo de un gradiente altitudinal [Composition and structure of the temperate forest of Santa Catarina Ixtepeji, Oaxaca, along an altitude gradient,Tesis de grado]. Oaxaca, Mexico: Universidad Nacional Autónoma de México; 2009. Spanish

[11] Suarez D. Formación de un corredor de hábitat de un bosque montano alto en un mosaico de páramo en el norte del Ecuador [Formation of a high montane forest habitat corridor in a páramo mosaic in northern Ecuador]. Lima, Perú: Universidad Nacional Agraria La Molina; 2009. Spanish

[12] Bhattarai K. Can Rapoport's rule explain tree species richness along the Himalayan elevation gradient, Nepal Diversity and Distributions [Internet]; 2006 [citado el 17 de agosto de 2018]. Disponible en: http://www.falk.vib.ne/nov/biodiversity. 